\title{
$A B$ INITIO CALCULATIONS OF PHONON DISPERSION RELATIONS IN ALUMINIUM
}

\author{
P. Scharoch ${ }^{a *}, \mathrm{~K}$. PARLIŃSKI ${ }^{b}$ AND A. KIEJNA ${ }^{c}$ \\ ${ }^{a}$ Institute of Physics, Wrocław University of Technology \\ Wyb. Wyspiańskiego 27, 50-370 Wrocław, Poland \\ ${ }^{b}$ Institute of Nuclear Physics, Radzikowskiego 152, 31-342 Kraków, Poland \\ 'Institute of Experimental Physics, University of Wrocław \\ Pl. M. Borna 9, 50-204 Wrocław, Poland
}

(Received November 9, 1999)

A direct method and $a b$ initio force constants were used to calculate phonon dispersion curves and phonon density in Al. The force constants were determined from the Hellmann-Feynman forces induced by the displacement of an atom in the $2 \times 2 \times 2$ fcc crystallographic supercell. This size of the supercell gives exact phonon frequencies at $\Gamma, X, L, W$ points of the Brillouin zone. The calculated phonon dispersion curves are in good agreement with the experimental data.

PACS numbers: $63.20 .-\mathrm{e}, 71.15 . \mathrm{Nc}$

The vibrational properties determine a wide range of macroscopic behaviour of solids, e.g. specific heat and the sound velocity. In addition, very low-frequency modes can be associated with phase transformations, while imaginary frequencies provide an indication that the calculated structure is not the most stable. Finally, the phonon spectrum enables a good approximation to free energies to be made via the quasiharmonic approximation. In view of these, derivation of phonons from $a b$ initio calculations has become a very important topic [1]. Generally the ab initio calculations of phonon frequencies fall into two methods: the linear response and the direct approach. Works still appear in the literature, in which various combinations of materials/methods/approximations and codes are tested. Aluminium is often considered to be a representative free-electron like metal and in recent years several calculations of phonons spectra for this material have been published. For example, the different approaches based on the phenomenological and the pseudopotential force constants have been compared in Ref. [2]. The ab initio cohesive energies were used to evaluate the phonon dispersion relations with and without three-body interaction [3]. Calculations based on the linear response theory which

*corresponding author; e-mail: scharoch@rainbow.if.pwr.wroc.pl 
enables to handle nonlocal pseudopotentials have been performed by Quong and Klein [4]. The phonon spectra calculated within the density-functional perturbation theory (DFPT) have been done by de Gironcoli [5]. One can observe that calculations based on the linear response theory, in case of $\mathrm{Al}$, show an excellent agreement with experiment. This is probably due to the fact that these methods are not limited by supercells (range of interaction taken into account). Another conclusion is that the local density approximation (LDA) works in this material very well. According to Ref. [3], taking into account three-body interaction improves considerably the results of calculations.

In this work the dispersion curves and frequency distribution of phonons are investigated by the direct/supercell method $[1,6]$, in which the cumulative force constants are calculated from ab initio Hellmann-Feynman (HF) forces, within LDA for the density functional, on the $2 \times 2 \times 2$ crystallographic supercell (which means that the interaction is taken into account up to the 5 th coordination shell).

The HF forces are defined as the minus derivative of the total energy $E_{\text {tot }}$ with respect to the position of ions $\boldsymbol{R}_{i}$,

$$
\boldsymbol{F}_{i}(n)=-\frac{\partial E_{\text {tot }}}{\partial \boldsymbol{R}_{i}(n)},
$$

where $n$ are the indices of the unit cell and $i$ is the Cartesian component. At the extremum all HF forces vanish. Non-zeroth HF forces arise, when a single atom $(m)$ is displaced by $u_{j}(m)$ from its equilibrium position. The arising forces are related to the cumulative force constants $\Phi_{i j}(n ; m)$ by the relation $[1,6]$

$$
\boldsymbol{F}_{i}(n)=-\sum_{m, j} \Phi_{i j}(n ; m) u_{j}(m)
$$

Cumulative force constants appear as a result of periodic boundary conditions imposed on the supercell. To calculate HF- forces an atomic configuration with a single displaced atom must be minimized with respect to the electronic part only. In case of the fcc crystallographic cell each such run provides 12 or $96 \mathrm{HF}$-force components for the $1 \times 1 \times 1$ or $2 \times 2 \times 2$ supercells, respectively. One run with positive and another with negative particle displacement along $z$ direction is usually performed. The data of HF forces $\boldsymbol{F}_{i}(n)$ and displacements $u_{j}(m)$ form an overdetermined set of equations, Eq. (2), for the force constants. This system is solved by the singular value decomposition algorithm $[6,7]$, which automatically provides a least squares solution. In case of the fcc lattice and the $2 \times 2 \times 2$ supercell, the 96 components of the HF forces lead to 13 non-zero independent parameters of the force constants. According to the direct method [1, 6, 8-10] the knowledge of the cumulative force constants allows one to define an approximate dynamical matrix, in which the summation over all atoms is confined to those atoms which reside within the volume of the supercell. The approximate dynamical matrix becomes equal to the conventional one at discrete wave vectors $k_{L}$ given by the equation $\exp \left(2 \pi \mathrm{i} k_{L} \cdot \boldsymbol{L}\right)=1[6]$, where $\boldsymbol{L}=\left(\boldsymbol{L}_{1}, \boldsymbol{L}_{2}, \boldsymbol{L}_{3}\right)$ are the lattice vectors of the supercell. At the wave vectors $k_{L}$ the phonon frequencies $\omega^{2}\left(k_{L}\right)$, calculated by diagonalization of the approximate dynamical matrix, are the same as those calculated from the exact dynamical matrix. At the fcc lattice and the $1 \times 1 \times 1$ supercell the "exact". wave vectors are at $\Gamma, X$ points, whereas at the $2 \times 2 \times 2$ 
supercell the "exact" wave vectors are at $\Gamma, X, L, W$, and two other points, namely, the midpoint between $\Gamma$ and $X$ along $\langle 100\rangle$ and $\langle 111\rangle$ directions. The advantage of the above described direct method is that it does not impose any limit to the range of interaction. When the supercell size is smaller than the range of interaction, the direct method interpolates the dispersion curves between the exact points.

In the reported calculations the $2 \times 2 \times 2$ supercell was used. The calculations were performed for few displacements (from $0.1 \%$ up to $5 \%$ ) to check for the convergence of dispersion curves and ensure that the calculations are within harmonic region. In the final runs the displacement $0.5 \%$ of the lattice constant was applied to calculate force fields. To increase the accuracy, two displacements in the opposite directions have been applied.

The equilibrium properties of $\mathrm{Al}$ crystal and $\mathrm{HF}$ forces have been calculated with the use of fhi $96 \mathrm{md}$ plane-wave code $[11,12]$. In the code the ion-electron interaction is represented by fully separable norm-conserving ab initio pseudopotentials $[13,14]$. The Kohn-Sham wave functions are expanded in the plane-wave basis set truncated at the kinetic energy cut-off $E_{\text {cut }}$. The integration in $k$-space is performed with the use of the Monkhorst-Pack scheme [15]. In our calculations the local density approximation (LDA) with the Ceperley-Alder form $[16,17]$ for the exchange-correlation energy has been applied. The correction accounting for the non-linear core-valence electron exchange-correlation interaction has been included into the pseudopotential.

The convergence of the phonon frequency with respect to computational parameters, the cut-off energy, and $k$-point sampling, was tested at the $L$ point of the Brillouin zone. A good convergence was observed starting from $E_{\text {cut }}=$ $25 \mathrm{Ry}$ and $10 \mathrm{k}$-points for the $2 \times 2 \times 2$ supercell. In the final calculations for the $2 \times 2 \times 2$ supercell $E_{\text {cut }}=30$ Ry and $20 k$-points were used. The influence of the Fermi-smearing parameter on the results was also checked. In agreement with [18], no significant difference was observed by its changing from $0.1 \mathrm{eV}$ to $0.2 \mathrm{eV}$ and the latter value was employed in the calculations. The results appeared to be influenced strongly (in particular the convergence of acoustic branches at $\Gamma$ point) by the accuracy in evaluation of the HF forces. Studying the stabilization of curves with respect to this parameter we managed to reduce its value to $1 \times 10^{-7}$ hartree/bohr.

The equilibrium bulk lattice constant of the fcc Al crystal has been determined by the total energy minimization, at the same supercell configuration and computational parameters as in further calculations of the HF force fields. The calculated lattice constant, $a=3.972 \AA$, is somewhat smaller than the corresponding experimental value (Table) which is due to the known deficiency of the LDA. However, the deviation amounts only about $1.9 \%$ so the agreement with experiment can be considered as good. The calculated bulk modulus is $816 \mathrm{kbar}$ which agrees well with the measured one (Table).

The calculated phonon dispersion curves, corresponding to the temperature $T=0 \mathrm{~K}$ are shown in Fig. 1. In the same figure they are compared with the experimental phonon frequencies measured by the inelastic neutron scattering [20]. The overall agreement of the shape of the curves is very good. The largest difference 
TABLE

Calculated lattice constant $a$ and bulk modulus $B$ for aluminium compared with other calculations and experimental data.

\begin{tabular}{c|c|c|c|c|c}
\hline \hline Property & \multicolumn{4}{|c|}{ Theory } & Experiment \\
\cline { 2 - 5 } & This work & $\begin{array}{c}\text { Pseudo- } \\
\text { potential [4] }\end{array}$ & LAPW [21] & DFPT [5] & \\
\hline$a[\AA]$ & 3.97 & 4.01 & 3.98 & 3.97 & $4.05[19]$ \\
$B[\mathrm{kbar}]$ & 816 & 740 & 839 & 783 & $783[22]$
\end{tabular}

appears at $X$ point, where the discrepancy between experiment and calculations of the transverse modes is about $6 \%$. However, in the majority of points the calculated frequencies lie within the experimental error. In Fig. 1 the error bars have been drawn for $X$ and $L$ points. A major qualitative difference between experiment and calculations is that experimental values of phonon frequencies at $X$ and $L$ points are almost equal whereas the calculated ones differ distinctly, by about $8 \%$.

The theory of lattice dynamics provides summation rules which follow from translational and rotational invariances of the crystal. At fcc lattice these invariances lead to one equation, which can be used to set the values of on-site force constant parameter. If this condition is satisfied the acoustic phonon branches at $k=0$ would point to $\omega=0$. Our dispersion curves, without imposed translational-

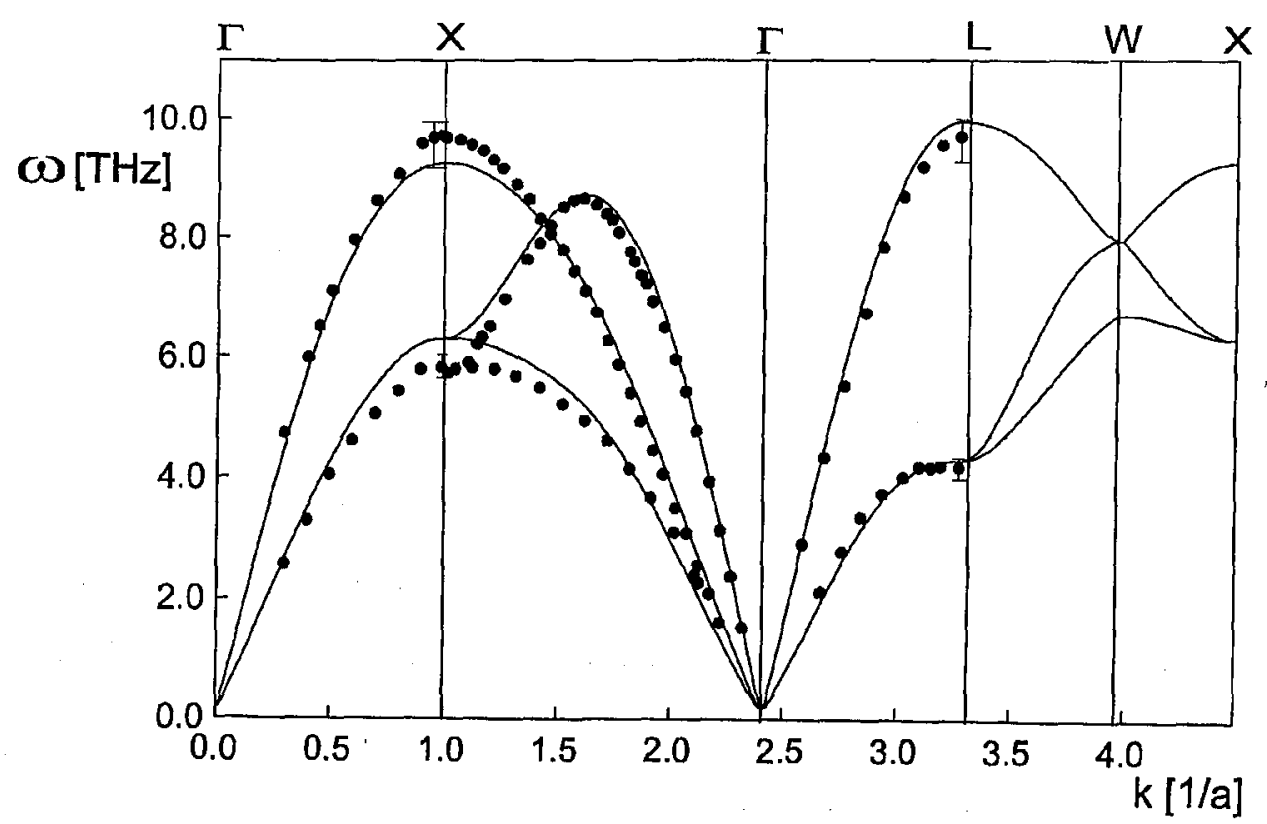

Fig. 1. Comparison of the calculated (solid lines) and experimental (circles) [20] phonon dispersion for Al. The wave vector is plotted in $2 \pi / a$ units where $a$ is given in $\AA$. 
-rotational invariances point at $k=0$ to $\omega=0.0 \pm 0.2 \mathrm{THz}$. (It varies with small changes in the lattice constant, but no regular behaviour was observed.) Since the invariance conditions should be satisfied within the $a b$ initio and direct methods, we did not correct our dispersion curves displayed in Fig. 1 for this effect.

Using Monte Carlo scheme based on the sampling of the dynamical matrix at many wave vectors distributed homogeneously over the Brillouin zone, one can calculate the phonon density function $g(\omega)$. The calculated density of states function, conventionally normalized to $\int g(\omega) \mathrm{d} \omega=1$, is shown in Fig. 2. The curve exhibits the most pronounced resonant behaviour at $\omega=8.8 \mathrm{THz}$, which in terms of energy expressed in units of Boltzmann constant corresponds to $T=422 \mathrm{~K}$.

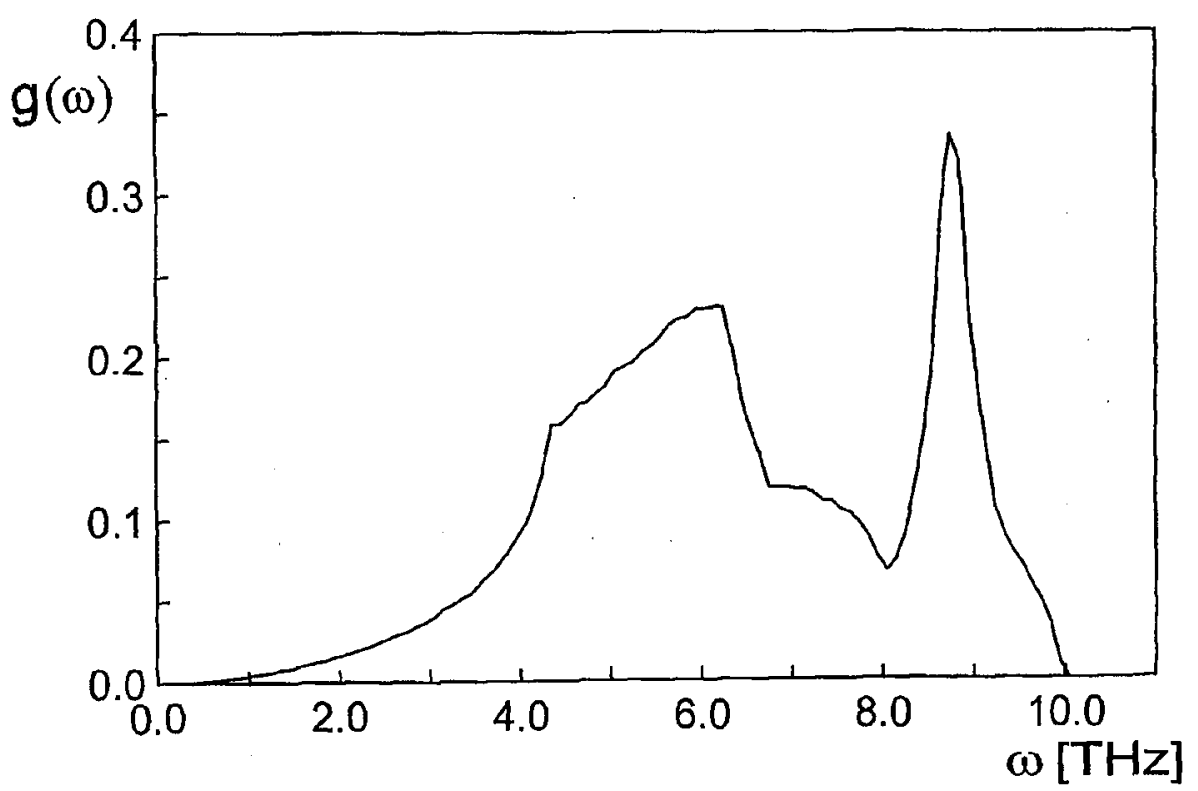

Fig. 2. The calculated phonon density of states in aluminium.

In summary, the density functional theory calculations of the lattice dynamics of aluminium have been presented. The calculated phonon dispersion curves show good agreement with experiment and other calculations. A major qualitative difference between experimental and calculated data is the lowering of the frequency at $X$ point in relation to $L$ point in the latter ones. According to the discussion at the beginning of the paper, the discrepancy between calculations and experiment can be attributed to both three-body interaction, not included in our calculations, as well as long-range interaction, neglected by the choice of supercell. The local density approximation, although it leads to underestimation of the lattice constant, seems to have less influence on phonon energies in aluminium. 


\section{Acknowledgments}

This work was partially supported by the Committee for Scientific Research, grant No. 2 PO3B 004 14. Most of the computations were performed in the Wrocław Supercomputer and Networking Centre (WCSS).

\section{References}

[1] G.J. Ackland, M.C. Warren, S.J. Clark, J. Phys., Condens. Matter 9, 7861 (1997).

[2] R.F. Wallis, A.A. Maradudin, V. Bortolani, A.G. Eguiluz, A.A. Quong, A. Franchini, G. Santoro, Phys. Rev. B 48, 6043 (1993).

[3] M. Li, S.J. Liu, N.X. Chen, Z. Phys. B 95, 297 (1994).

[4] A.A. Quong, B.M. Klein, Phys. Rev. B 46, 10734 (1992).

[5] S. de Gironcoli, Phys. Rev. B 51, 6773 (1995).

[6] K. Parliński, Z.Q. Li, Y. Kawazoe, Phys. Rev. Lett. 78, 4063 (1997).

[7] W.H. Press, B.P. Flamery, S.A. Teukolsky, W.T. Vetterling, Numerical Recipes, Cambridge University Press, Cambridge 1988, p. 301.

[8] W. Frank, C. Elsässer, M. Fähnle, Phys. Rev. Lett. 74, 1791 (1995).

[9] G. Kresse, J. Furthmüller, J. Hafner, Europhys. Lett. 32, 729 (1995).

[10] M.H.F. Sluiter, M. Weinert, Y. Kawazoe, Europhys. Lett. 43, 183 (1998).

[11] M. Bockstedte, A. Kley, J. Neugebauer, M. Scheffler, Comput. Phys. Commun. 107, 187 (1997).

[12] J. Neugebauer, M. Scheffler, Phys. Rev. B 46, 16067 (1992).

[13] L. Kleinman, D.M. Bylander, Phys. Rev. Lett. 48, 1425 (1982).

[14] D.R. Hamann, Phys. Rev. B 40, 2980 (1989).

[15] H.J. Monkhorst, J.D. Pack, Phys. Rev. B 13, 5188 (1976).

[16] D.M. Ceperley, B.J. Alder, Phys. Rev. Lett. 45, 566 (1980).

[17] J.P. Perdew, A. Zunger, Phys. Rev. B 23, 5048 (1981).

[18] F. Wagner, Th. Laloyaux, M. Scheffler, Phys. Rev. B 57, 2102 (1998).

[19] B. Chakraborty, R.W. Siegel, Phys. Rev. B 27, 4535 (1983).

[20] R. Stedman, G. Nilsson, Phys. Rev. 145, 492 (1966).

[21] A. Khein, D.J. Singh, C.J. Umrigar, Phys. Rev. B 51, 4105 (1995).

[22] K.A. Gschneidner, Jr., in: Solid State Physics, Eds. H. Ehrenreich, F. Seitz, D. Turnbull, Vol. 16, Academic, New York 1964, p. 275. 